\title{
Impact of myo-inositol treatment in women with polycystic ovary syndrome in assisted reproductive technologies
}

\author{
Philippe Merviel ${ }^{1^{*}} \mathbb{B}$, Pandora James ${ }^{1}$, Sarah Bouée ${ }^{1}$, Mathilde Le Guillou', Camille Rince ${ }^{1}$, \\ Charlotte Nachtergaele ${ }^{2}$ and Véronique Kerlan²
}

\begin{abstract}
Polycystic ovary syndrome (PCOS) is marked in 30 to $40 \%$ by insulin resistance and hyperandrogenism. Myo-inositol (MI) increases insulin sensitivity, decreases hyperandrogenism and improves the menstrual cycle. Its effect during assisted reproductive technologies (ART) has been studied by many authors. We conducted a review of the literature on the impact of $\mathrm{MI}$ administration in PCOS women in assisted reproductive technologies. Myo-inositol is effective in normalizing ovarian function, improving oocyte and embryo quality in PCOS, however further evaluations by large multicentre randomized controlled trials are needed to assess the clinical pregnancy and live birth rates in ART.
\end{abstract}

Keywords: Polycystic ovary syndrome (PCOS), Myo-inositol, In vitro fertilization, Intracytoplasmic sperm injection (IVF-ICSI), Embryo quality, Pregnancy

\section{Plain English summary}

We conducted a review of the literature on the impact of myo-inositol (MI) administration in polycystic ovary syndrome (PCOS) women in assisted reproductive technologies (ART). MI is effective in normalizing ovarian function, improving oocyte and embryo quality in PCOS, however further evaluations by large multicentre randomized controlled trials are needed to assess the clinical pregnancy and live birth rates in ART.

\section{Introduction}

Polycystic ovary syndrome (PCOS) is the most common cause of ovulation disorders, hyperandrogenism and infertility due to ovulatory dysfunction, affecting more than $7 \%$ of childbearing age women [1]. PCOS is associated with obesity in $80 \%$ of cases and is associated with a metabolic syndrome with insulin resistance in $30-40 \%$

*Correspondence: philippe.merviel@chu-brest.fr

${ }^{1}$ Fertility and ART Department, Brest University Hospital, 2 Avenue Foch, 29200 Brest, France

Full list of author information is available at the end of the article of cases, which can aggravate PCOS [2]. Hyperglycemia inhibits hepatic production of Sex Hormone Binding Globulin (SHBG), which leads to an increase of free androgens in the blood circulation, and insulin resistance increases the production of androgens by the theca cells. The management of this insulin resistance is therefore essential in the treatment of PCOS, and is based on nutritional rules, physical activity and other molecules including myo-inositol (MI). The prescription of insulinsensitizing agents such as metformin is indicated only in cases of glucose intolerance and type 2 diabetes mellitus. In this paper, we aimed to review the role of myo-inositol, a natural insulin sensitizer, on menstrual cycle disorders, ovulation induction and in vitro fertilization/intracytoplasmic sperm injection (IVF/ICSI) outcomes in women with PCOS.

\section{Mechanism of action of myo-inositol}

Inositol is a polyalcohol of which there are nine stereoisomers (cyclohexane-1,2,3,4,5,6-hexol). Two of them have been shown to mediate the post-receptor effects of insulin: myo-inositol (MI-cis-1,2,3,5-trans-4,6-cyclohexanehexol) original author(s) and the source, provide a link to the Creative Commons licence, and indicate if changes were made. The images or other third party material in this article are included in the article's Creative Commons licence, unless indicated otherwise in a credit line to the material. If material is not included in the article's Creative Commons licence and your intended use is not permitted by statutory regulation or exceeds the permitted use, you will need to obtain permission directly from the copyright holder. To view a copy of this licence, visit http://creativecommons.org/licenses/by/4.0/. The Creative Commons Public Domain Dedication waiver (http://creativeco mmons.org/publicdomain/zero/1.0/) applies to the data made available in this article, unless otherwise stated in a credit line to the data. 
and D-chiro-inositol (DCI-cis-1,2,4-trans-3,5,6-cyclohexanehexol) (DCI). The food categories that contain the highest concentration of inositols are fruits, beans, corn and nuts. DCI negatively interferes with MI absorption at the intestinal level. Uptake of free inositol by tissues occurs by a membrane dependant sodium inositol cotransporter, for which MI has 10 times greater affinity than DCI. MI and DCI are mediated by some inositolphosphoglycans (IPGs), already known as second messengers. These mediators are then internalized and modify enzymatic activity and intracellular metabolism, mimicking the action of insulin. When insulin binds to its receptor, these IPGs are generated by hydrolysis of glycosylphosphatidylinositol (GPI) lipids and/or specific proteins located on the outer part of the cell membrane. Two IPGs are formed: IPG-DCI (or IPG-P) and IPG-MI (or IPG-A). IPG-P will directly activate the glycogen synthase but will also indirectly activate it via the activation of phosphoprotein phosphatase 1 (PP1). IPG-A causes direct glucose uptake and inhibits cAMP protein kinase $\mathrm{A}$ and adenylate cyclase, thereby activating PP1. These effects allow a decrease in blood glucose levels (insulinlike effect), regardless of the signal passing through the insulin receptor [3]. In women with PCOS, impaired inositol and/or GPI metabolism contributes to insulin resistance, but obesity plays a specific role in abnormal IPG-P production independently of PCOS [3]. MI decreases body weight, leptin secretion and increases HDL cholesterol [4], but this author have noted that metabolic risk factor benefits of inositol treatment were not observed in the morbidly obese subgroup of women. Thanks to its antioxidant action (SOD, catalase and GSH increase), MI improves cell morphology and growth, as well as the synthesis of lipids participating in cell membranes. Figure 1 summarizes the different actions of MI in the ovary.

Myo-inositol is the most abundant inositol isomer in the human body; DCI is synthesized by an insulindependent epimerase that converts MI into DCI. Epimerase activity dysregulation affects $\mathrm{MI} / \mathrm{DCI}$ ratio, as in PCOS where a defect of MI utilization could impair FSH and insulin signaling. Each organ has a specific MI/DCI ratio related to its function [5]. Therefore, in glycogen

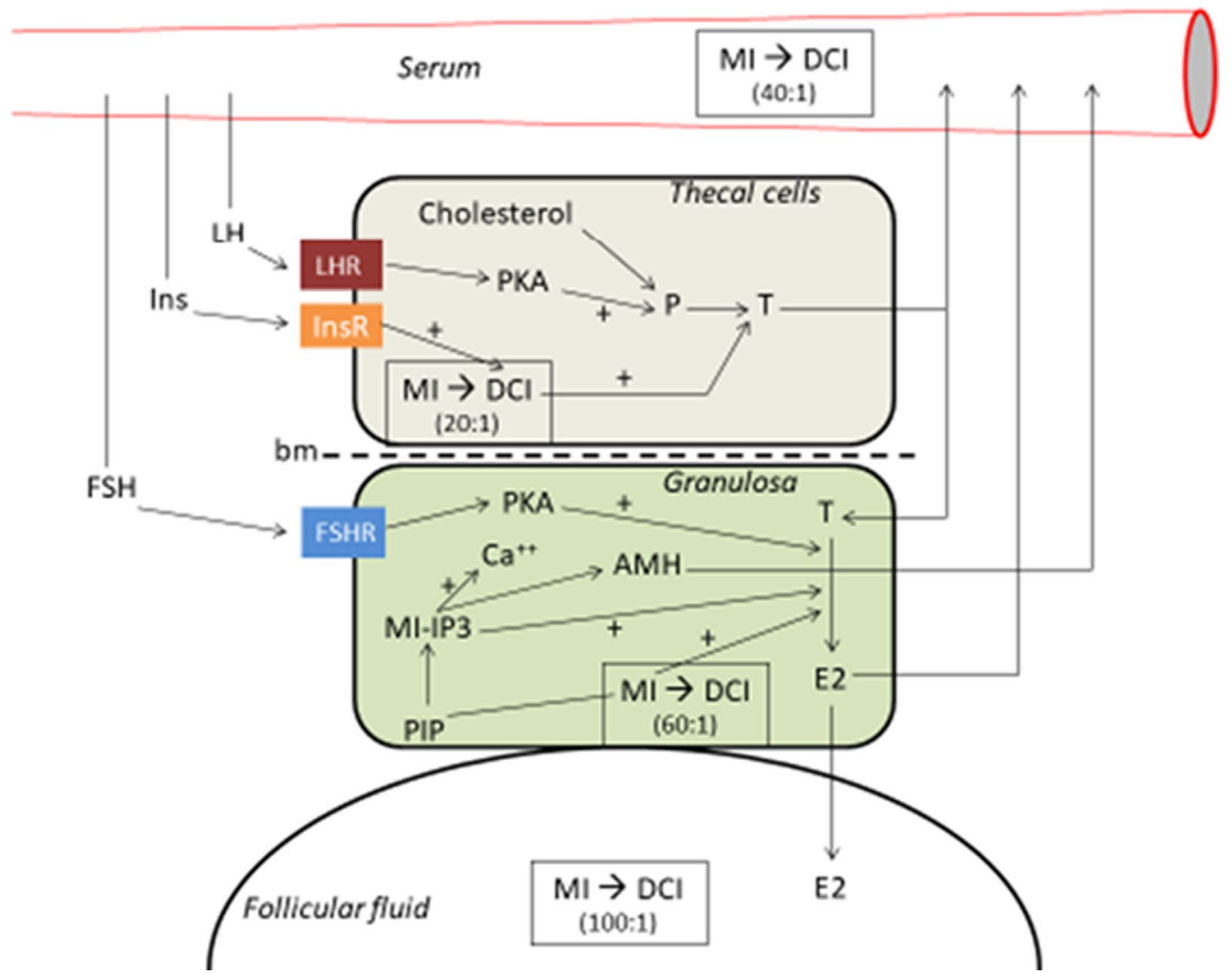

Fig. 1 Roles of myo-inositol (MI) in the ovary (original figure from the author, after review of patho-physiologic effects of MI, DCl and others hormones on ovarian cells). MI: myo-inositol; DCI: D-chiro-inositol; (40:1): MI/DCI ratio; LHR: LH receptor; PKA: protein kinase A; P: progesterone; T: testosterone; INs: insulin; InsR: insulin receptor; bm: basalis membrane; PIP: phospho inositide phosphate; IP3: inositide triphosphate; AMH: anti-Müllerian hormone; E2: estradiol; + : stimulating effect 
storage organs, high levels of DCI have been observed. In the ovary, $\mathrm{DCI}$ is responsible for an excess production of insulin-dependent testosterone, whereas MI enhances the action of FSH, via anti-Müllerian hormone (AMH). MI has been found in follicular fluid [2] and appears to improve oocyte and embryo quality. Usually, the MI/ DCI ratio is 100:1, whereas in PCOS it is 0.2:1 [6]. When the concentration of $\mathrm{MI}$ is reduced in the follicular fluid (which is the case of PCOS, where it is reduced by 500 times), epimerase activation is excessive leading to an excess of DCI, an increase in insulin resistance and an increase in LH levels. If DCI concentrations above the MI/DCI limit ratio of 70:1 in follicular fluid, the blastocyst quality was decreased. The adequate MI/DCI ratio for supplementation is 40:1. This ratio is the best (among seven different ratios between $\mathrm{MI}$ and $\mathrm{DCI}$ ) for PCOS therapy aimed at restoring menstrual cycle and ovulation, increasing progesterone and SHBG and decreasing $\mathrm{LH}$, testosterone and insulin levels [7].

\section{Effects of MI on menstrual cycle disorders}

In PCOS, early follicular growth is excessive, but subsequent progression to a dominant follicle is interrupted (follicular arrest). Intraovarian androgens have been implicated in the excess of follicles and the elevated serum estradiol levels. This increased production of androgens is an inherent property of thecal cells, but it is increased by the surplus of LH and by hyperinsulinism. In women with PCOS, treatment with metformin (MET) ameliorated the insulin sensitivity and decreased the androgens levels, but the limitations to MET use are its gastrointestinal side effects. In this case of PCOS, the place of MI was evaluated. Studies by Zacché et al. [8] and Minozzi et al. [9] show that MI leads to a decrease in $\mathrm{LH}$ and androgen levels, as well as a decrease in insulin resistance. Thus, MI is believed to be able to re-establish ovulatory menstrual cycles (especially in obese women with PCOS) but its effect on pregnancy rates is difficult to determine (different diagnoses, insufficient power of studies, non-comparative studies). The second anomaly is the failure to select a dominant follicle, leading to the accumulation of selectable follicles and the typical aspect of polycystic (multifollicular) ovaries when ultrasonography is performed. This phenomenon called follicular arrest is the result of a lack of FSH action and/or premature LH action. Studies have shown the role played by anti-Müllerian hormone (AMH) in inhibiting the follicular response to FSH [1]. Hyperinsulinism, on the other hand, increases the sensitivity of follicles to LH. MI is responsible for a decrease in $\mathrm{LH}$, in the $\mathrm{LH} / \mathrm{FSH}$ ratio and in testosterone and androstenedione. When ovulation is induced in PCOS women with hyperinsulinism, MI reduces the risk of multifollicular development.
Therefore, MI reduces androgen levels (testosterone and androstenedione), corrects the LH/FSH ratio, restores normal menstrual cycles and induces ovulation, thereby facilitating spontaneous pregnancies by adequate luteal phase progesterone production [10].

\section{Impact of $\mathrm{MI}$ in IVF/ICSI}

The role of MI and/or DCI supplementation in women with PCOS undergoing assisted reproductive technologies (ART) to improve oocyte quality, embryo quality and chances to achieve pregnancy has been investigated [1113]. However, a recent meta-analysis focused on women with PCOS undergoing ICSI found inconclusive evidence on MI efficacy [14]. Table 1 summarizes the main randomized studies on the impact of Myo-inositol (MI), alone or in combination, for induction, ovarian stimulation, or IVF/ICSI in women with PCOS. MI increases the sensitivity of polycystic ovaries to gonadotropins, leading to a reduction in the doses of FSH required:-500 IU for Lagana et al. [15],-327 IU for Zheng et al. [16] (p: 0.007); whereas the opposite is observed for DCI (Carlomagno paradox [17]). MI decreases estradiol levels on the day of ovulation trigger, reduces the number of intermediate-sized follicles and increases the number of large follicles (without increasing the total number of oocytes retrieved), thereby contributing to a reduction of the risk of ovarian hyperstimulation. There is an improvement in oocyte quality and oocyte maturation, an increase in cleavage rate, embryo development (expanded blastocyst) and quality, and an increase in the pregnancy rate in women with PCOS [12]. Chiu et al. [2] demonstrated the link between the concentration of MI in the follicular fluid and oocyte and embryo quality in 53 women with a normal response. He recovered 60 follicular fluids, 38 containing a mature oocyte which was then fertilized (group A) and 22 containing an immature, non-fertilized oocyte (group B). The follicular volume and MI concentration were significantly higher in group A. Significant positive correlations were found between the intrafollicular concentration of MI and the level of estradiol in the follicular fluid, the cleavage rate of fertilized oocytes, the stage of embryos ( \pm 4 cells) and embryo quality (grade). The same author showed that MI supplementation is associated to meiotic progression of mouse germinal vesicle oocytes, enhancing intracellular $\mathrm{Ca}^{2+}$ oscillation and leading to the end of meiosis [18]. Sene et al. [19] performed a randomized controlled trial comparing two groups of 25 women with PCOS, one group receiving $4 \mathrm{~g}$ of $\mathrm{MI}+400 \mathrm{mg}$ of folic acid per day, the other receiving folic acid alone. These pre-treatments started 1 month prior to the start of a GnRH + FSH antagonist protocol, and were continued until the morning of ovulation triggering (via $\mathrm{GnRH}$ agonist therapy). The percentage of 


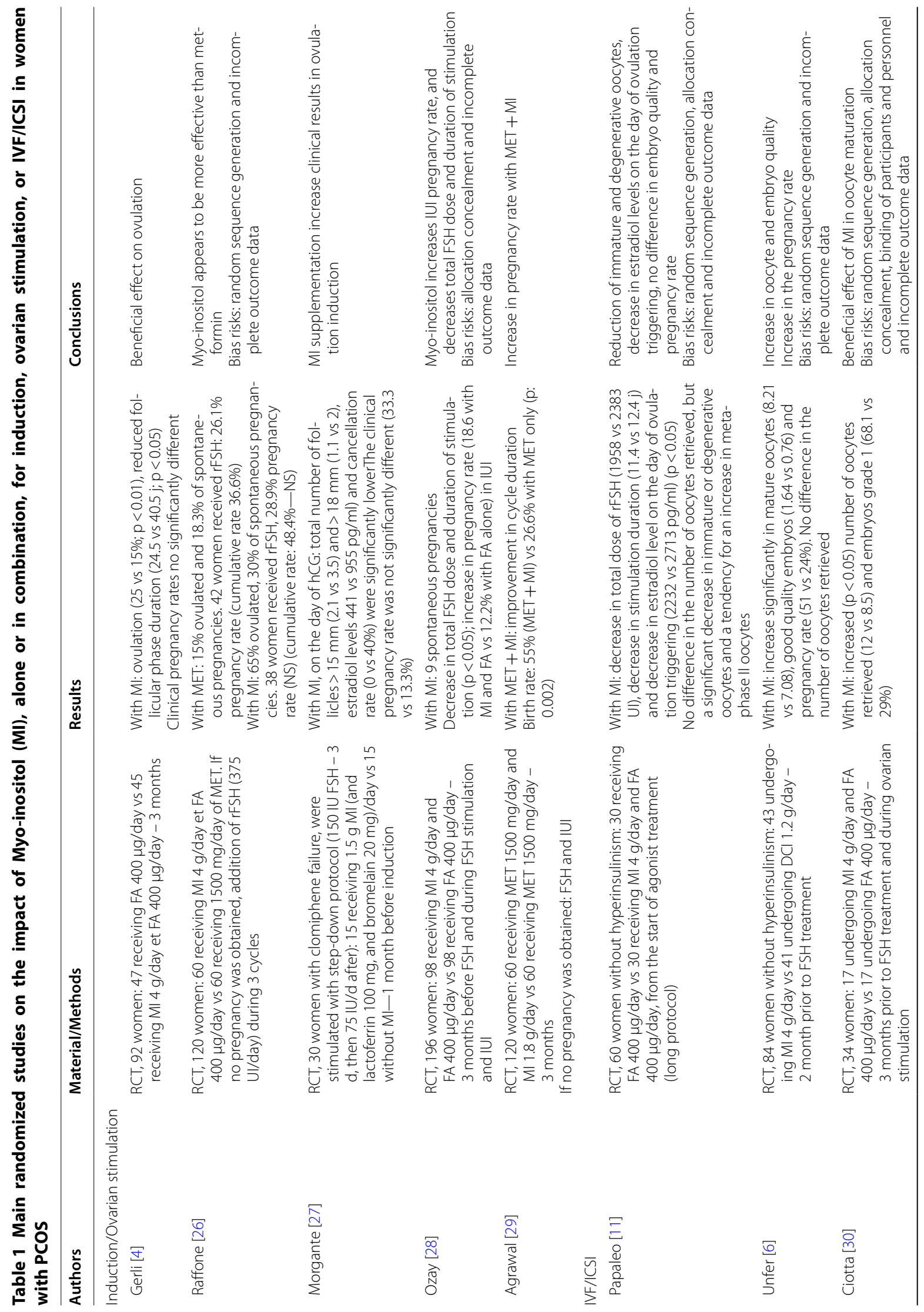




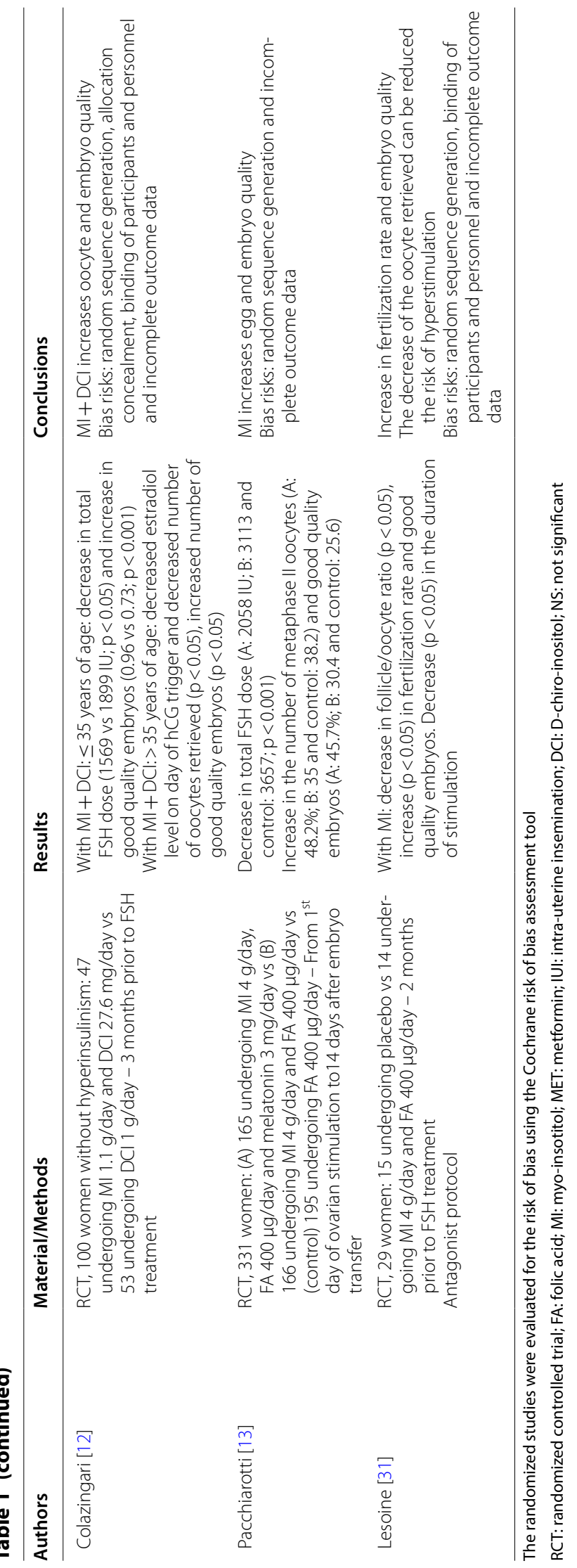


metaphase II oocytes (78 vs 58\%; p: 0.003 ), the fertilization rate (65 vs 46\%; p: 0.03 ) and the percentage of good quality embryos (p: 0.006) were significantly higher in the $\mathrm{MI}+$ folic acid group. While the expression in the granulosa cells of three genes involved in oocyte quality (PGK1, RGS2 and CDC42) was found to be significantly increased in the $\mathrm{MI}+$ folic acid group, no significant difference was reported in the concentration of reactive oxygen species (ROS) in the follicular fluid, suggesting that the effect of MI on oocyte quality is independent of its antioxidant action. In this study, no effect was observed concerning the cumulative pregnancy rate (40 vs $35 \%$ respectively), contrasting with the study by Artini et al. [20] which found an increase in the pregnancy rate (60 vs $32 \%$; $\mathrm{p}<0.05$ ).

Two meta-analyses have been published confirming the impact of MI among women with PCOS in IVF/ICSI. Lagana et al. [15] studied the total gonadotropin dose and duration of stimulation in PCOS and non-PCOS women with $\mathrm{MI}$ and DCI. Intervention group received $4 \mathrm{~g}$ of MI in six studies, $2 \mathrm{~g}$ of MI in the Artini' study [20], and $1.1 \mathrm{~g}$ $\mathrm{MI}+27.6 \mathrm{mg}$ DCI in the Colazingari' study [12]. Comparator was placebo in six studies $(400 \mu \mathrm{g}$ of folic acid in five studies), $1.2 \mathrm{~g}$ of DCI in the Unfer' study [6] and $1 \mathrm{~g}$ of DCI in the Artini' study [20]. The period of administration was variable, between the day of GnRH agonist administration [11] to $12[12,13]$ or 8 weeks before rFSH administration for Unfer et al. [6]. He reported an effect of MI supplementation on both endpoints in women with PCOS, but only on the total dose in non-PCOS women. The difference is significant between MI and DCI for the decrease in total gonadotropin dose $(1953 \pm 397$ vs. $2360 \pm 301 \mathrm{IU}, \mathrm{p}<0.01$ ) and duration of stimulation $(11.1 \pm 0.8$ vs $12.7 \pm 1.1$ days, $\mathrm{p}<0.01)$, for increased oocyte maturity $(8.21 \pm 2.39$ vs $7.08 \pm 2.67$ metaphase II oocytes, $\mathrm{p}<0.05)$ and clinical pregnancy rate $(22 \mathrm{vs}$ $11 \%, \mathrm{p}<0.05)$. No effect is observed on cancelled cycles and on the total number of oocytes retrieved. Zheng et al. [16] conducted a meta-analysis of 6 studies (913 PCOS), showing an increase in clinical pregnancy rates after pre-treatment with MI: 33.3 vs $27.6 \%$, i.e. OR: 1.45 (95\% CI 1.04-1.96, p: 0.03). This author reports a $27 \%$ decrease in miscarriages ( $95 \%$ CI $0.08-0.50$, p: 0.0006). In addition, the proportion of grade 1 embryos is significantly increased, and the number of germinal vesicle, and degenerated oocytes retrieved and the total dose of gonadotropins are significantly reduced. No differences were found in the total number of oocytes, in oocyte maturity, the duration of stimulation or the serum estradiol level on the day of ovulation triggering.

On the contrary, three meta-analyses failed to conclude on the effect of MI in women with PCOS [14, 21, 22]. Mendoza [14] reported, in 8 studies involving 1019 PCOS women, a not significant trend towards improvement in egg quality (OR: 2.2 ; 95\% CI $0.8-5.8$ ), embryo quality (OR 1.6; 95\% CI 0.3-6.7) and pregnancy rate (OR: 1.2; 95\% CI 0.8-1.8) with MI administration. He concluded that future studies of dose, size and duration of DCI are necessary. Since, two other controlled, randomized, double blind parallel group studies of the same author [21, 22] showed (after 12 weeks of treatment in women with PCOS undergoing ICSI), (i) a significant increase of pregnancy and live birth rates (65.5 vs. 25.9; p: 0.003 , and 55.2 vs. 14.8 ; p: 0.002 , respectively) and a decrease of ovarian hyperstimulation syndrome (3.4 vs. $18.5 \%$; p: 0.07 ) with a 3.6:1 MI/DCI ratio compared to a $40: 1$ ratio [21]; (ii) a positive influence (p: 0.006 ) on the quality of the cytoplasm of the oocyte with a 1.8:1 ratio compared to a $20: 1$ ratio [22]. So, the debate on the appropriate MI/DCI ratio remains unresolved. In the Bhide' meta-analysis [23] (18 trials included) the primary outcome (changes in antiMüllerian hormone and antral follicle count before and after treatment), any conclusion was suitable. For the secondary outcomes, no significant differences between $\mathrm{MI} / \mathrm{DCI}$ and control group were reported on number of oocytes, metaphase II oocytes, top grade embryos, clinical pregnancy rate and risk of ovarian hyperstimulation syndrome. This author noted the very low quality of these studies. In the Cochrane Database analysis [24] (11 trials involving 1472 women with PCOS and IVF), no pooled evidence is available for use of MI versus placebo, insulin-sensitizing and ovulation induction agents for women with PCOS undergoing pre-treatment to IVF. It is unable to show that a myo-inositol treatment increases the chances of pregnant and having a baby, and unclear on whether MI could lower miscarriage rates. In this metaanalysis, the author regretted the small number and the poor quality of the trials, with serious risk of bias associated with poor reporting of methods, imprecision and inconsistency.

Therefore, MI administered 3 months prior to the start of ovarian stimulation, reduces the doses of FSH required for the follicular response, lowers the estradiol level on the day of ovulation triggering, thus reducing the risk of ovarian hyperstimulation [25] and the number of cancelled cycles. Meanwhile, oocyte and embryo quality is increased.

\section{Conclusions}

MI, at a dose of $4 \mathrm{~g}$ per day ( $2 \mathrm{~g}$ twice per day), three months prior to ovarian stimulation, is effective in normalizing ovarian function, improving oocyte and embryo quality in PCOS. However, further evaluations by large multicentre randomized controlled trials are needed to assess the clinical pregnancy and live birth rates in ART, because many published studies were heterogeneous. 
In addition, myo-inositol is a secure and cost-effective alternative in the treatment of PCOS, with no side effects observed in the standard dosage.

\begin{abstract}
Abbreviations
PCOS: Polycystic ovarian syndrome; SHBG: Sex-hormone binding protein; MI: Myo-inositol; IVF: In vitro fertilization; ICSI: Intracytoplasmic sperm injection; DCI: D-chiro-inositol; IPG: Inositolphosphoglycan; GPI: Glycosylphosphatidylinositol; PP1: Phosphoprotein phosphatase 1; HDL: High density level; SOD: Superoxide dismutase; GSH: Glutathione; FSH: Folliculostimulating hormone; AMH: Anti-Müllerian hormone; LH: Luteinizing hormone; MET: Metformin; ART : Assisted reproductive technology; $\mathrm{Ca}^{++}$: Calcium ion; $\mathrm{GnRH}$ : Gonadotropin releasing hormone; ROS: Reactive oxygen species; OR: Odds-ratio; 95\% Cl: Confidence interval at 95.
\end{abstract}

\section{Acknowledgements}

The authors thank Pandora James for their comments, suggestions and critical reading of the manuscript.

\section{Authors' contributions}

PM: substantial contributions to the conception, design of the work, the acquisition, analysis and interpretation of data; have drafted the work or substantively revised it (Head of the Fertility and ART department). PJ: The acquisition of data. SB: the acquisition, analysis and interpretation of data. MLG: the acquisition of data. CR: the acquisition of data. CN: the acquisition of data. VK: design of the work, the acquisition, analysis and interpretation of data; have drafted the work or substantively revised it (Head of the Endocrinology and Metabolism department). Each author have approved the submitted version and have agreed both to be personally accountable for the author's own contributions and to ensure that questions related to the accuracy or integrity of any part of the work, even ones in which the author was not personally involved, are appropriately investigated, resolved, and the resolution documented in the literature. All authors read and approved the final manuscript.

\section{Funding}

Not applicable.

\section{Availability of data and materials}

The material contained in this manuscript has not been published, has not been submitted or is not being submitted elsewhere. The datasets used and/ or analysed during the current study are available from the corresponding author on reasonable request.

\section{Ethical approval and consent to participate}

Not applicable (review of the literature).

\section{Consent for publication}

The manuscript has been read and approved by all authors.

\section{Competing interests}

The authors report no conflicts of interest in relation to the present study.

\section{Author details}

1 Fertility and ART Department, Brest University Hospital, 2 Avenue Foch, 29200 Brest, France. ${ }^{2}$ Endocrinology and Metabolism Department, Brest University Hospital, Boulevard Tanguy Prigent, 29200 Brest, France.

Received: 16 September 2020 Accepted: 5 January 2021

Published online: 19 January 2021

\section{References}

1. Merviel P, Bouée S, Ménard M, Le Martelot MT, Roche S, Lelièvre C, et al. Which ovarian stimulation to which women: the polycystic ovary syndrome (PCOS). Gynecol Obstet Fertil Senol. 2017;45:623-31.

2. Chiu TTY, Rogers MS, Law ELK, Britton-Jones CM, Cheung LP, Haines CJ. Follicular fluid and serum concentrations of myo-inositol in patients undergoing IVF : relationship with oocyte quality. Hum Reprod. 2002;17:1591-6.

3. Lagana AS, Garzon S, Casarin J, Franchi M, Ghazzi F. Inositol in polycystic ovary syndrome: restoring fertility through a pathophysiology-based approach. Trends Endocrinol Metab. 2018:29:768-80.

4. Gerli S, Papaleo E, Ferrari A, Di Renzo GC. Randomized, double-blind placebo-controlled trial: effects of myo-inositol on ovarian function and metabolic factors in women with PCOS. Eur Rev Med Pharmacol Sci. 2007:11:347-54.

5. Larner J. D-chiro-inositol - its functional role in insulin action and its deficit in insulin resistance. Int J Exp Diabetes Res. 2002;3:47-60.

6. Unfer V, Carlomagno G, Rizzo P, Raffone E, Roseff S. Myo-inositol rather than D-chiro-inositol is able to improve oocyte quality in intracytoplasmic sperm injection cycles: a prospective, controlled, randomized trial. Eur Rev Med Pharm Sci. 2011;15:452-7.

7. Nordio M, Basciani S, Camajani E. The 40:1 myo-inositol/D-chiro-inositol plasma ratio is able to restore ovulation in PCOS patients: comparison with other ratios. Eur Rev Med Pharmacol Sci. 2019:23:5512-21.

8. Zacche MM, Caputo L, Filippis S, Zacche G, Dindelli M, Ferrari A. Efficacy of myo-inositol in the treatment of cutaneous disorders on young women with polycystic ovary syndrome. Gynecol Endocrinol. 2009;25:508-13.

9. Minozzi M, Andrea GD, Unfer V. Treatment of hirsutism with myo-inositol : a prospective clinical study. Reprod Biomed Online. 2008;17:579-82.

10. Papaleo E, Unfer V, Baillargeon JP, De Santis L, Fusi F, Brigante C. Myoinositol in patients with polycystic ovary syndrome : a novel method for ovulation induction. Gynecol Endocrinol. 2007;23:700-3.

11. Papaleo E, Unfer V, Baillargeon JP, Fusi F, Occhi F, De Santis L. Myo-inositol may improve oocyte quality in intracytoplasmic sperm injection cycles: a prospective, controlled, randomized trial. Fertil Steril. 2009;91:1750-4.

12. Colazingari S, Treglia M, Najjar R, Bevilacqua A. The combined therapy myo-inositol plus D-chiro-inositol, rather than D-chiro-inositol, is able to improve IVF outcomes : results from a randomized controlled trial. Arch Gynecol Obstet. 2013;288:1405-11.

13. Pacchiarotti A, Carlomagno G, Antonini G, Pacchairotti A. Effect of myoinositol and melatonin versus myo-inositol, in a randomized controlled trial, for improving in vitro fertilization of patients with polycystic ovarian syndrome. Gynecol Endocrinol. 2016;32:69-73.

14. Mendoza N, Perez L, Simoncini T, Genazzani A. Inositol supplementation in women with polycystic ovary syndrome undergoing intracytoplasmic sperm injection : a systematic review and meta-analysis of randomized controlled trials. Reprod Biomed Online. 2017;35:529-35.

15. Lagana AS, Vitagliano A, Noventa M, Ambrosini G, D'Anna R. Myo-inositol supplementation reduces the amount of gonadotropins and length of ovarian stimulation in women undergoing IVF : a systematic review and meta-analysis of randomized controlled trials. Arch Gynecol Obstet. 2018:298:675-84.

16. Zheng $X$, Lin D, Zhang Y, Lin Y, Song J, Li S, et al. Inositol supplement improves clinical pregnancy rate in infertile women undergoing ovulation induction for ICSI or IVF-ET. Medicine. 2017;96(49):e8842.

17. Carlomagno G, Unfer V, Roseff S. The D-chiro_inositol paradox in the ovary. Fertil Steril. 2011:95:2515-6.

18. Chiu TTY, Rogers MS, Briton-Jones C, Haines C. Effects of myo-inositol on the in-vitro maturation and subsequent development of mouse oocytes. Hum Reprod. 2003;18:408-16.

19. Sene AA, Tabatabaie A, Nikniaz H, Alizadeh A, Sheibani K, Alisaraie MM, et al. The myo-inositol effect on the oocyte quality and fertilization rate among women with polycystic ovary syndrome undergoing assisted reproductive technology cycles : a randomized clinical trial. Arch Gynecol Obstet. 2019;299:1701-7.

20. Artini PG, Di Berardino OM, Papini F, Genazzani AD, Simi G, Ruggiero M, et al. Endocrine and clinical effects of myo-inositol administration in polycystic ovary syndrome: a randomized study. Gynecol Endocrinol. 2013;29:375-9.

21. Mendoza N, Diaz-Ropero MP, Aragon M, Maldonado V, Llaneza P, Lorente $J$, et al. Comparison of the effect of two combinations of myo-inositol and D-chiro-inositol in women with polycystic ovary syndrome undergoing ICSI: a randomized controlled trial. Gynecol Endocrinol. 2019;35:695-700.

22. Mendoza N, Galan MI, Molina C, Mendoza-Tesarik R, Conde C, Mazheika $\mathrm{M}$, et al. High dose of d-chiro-inositol improves oocyte quality in women with polycystic ovary syndrome undergoing ICSI: a randomized controlled trial. Gynecol Endocrinol. 2020;36:398-401. 
23. Bhide P, Pundir J, Gudi A, Shah A, Homburg R, Acharya G. The effect of myo-inositol/di-chiro-inositol on markers of ovarian reserve in women with PCOS undergoing IVF/ICSI: a systematic review and meta-analysis. Acta Obstet Gynecol Scand. 2019;98:1235-44.

24. Showell MG, Mackenzie-Proctor R, Jordan V, Hodgson R, Farquhar C. Inositol for subfertile women with polycystic ovary syndrome. Cochrane Database Syst Rev. 2018;8:012378.

25. Turan GA, Eskicioglu F, Sivrikoz N, Cengiz H, Adakan S, Gur EB, et al. Myoinositol is a promising treatment for the prevention of ovarian hyperstimulation syndrome (OHSS): an animal study. Arch Gynecol Obstet. 2015;292:1163-71.

26. Raffone E, Rizzo P, Benedetto V. Insulin sensitiser agents alone and in cotreatment with r-FSH for ovulation induction in PCOS women. Gynecol Endocrinol. 2010;26:275-80.

27. Morgante G, Orvieto R, Di Sabatino A, Musacchio MC, De Leo V. The role of inositol supplementation in patients with polycystic ovary syndrome, with insulin resistance, undergoing the low-dose gonadotropin ovulation induction regimen. Fertil Steril. 2011:95:2642-4.

28. Ozay OE, Ozay AC, Cagliyan E, Okyay RE, Gulekli B. Myo-inositol administration positively effects ovulation induction and intrauterine insemination in patients with polycystic ovary syndrome: A prospective, controlled, randomized trial. Gynecol Endocrinol. 2017;33:524-8.

29. Agrawal A, Mahey R, Kachhawa G, Khadgawat R, Vanamail P, Kriplani A. Comparison of metformine plus myo-inositol vs metformine alone in PCOS women undergoing ovulation induction cycles : Randomized controlled trial. Gynecol Endocrinol. 2019;35:511-4

30. Ciotta L, Stracquadanio M, Pagano I, Carbonaro A, Palumbo M, Gulino F. Effects of myo-inositol supplementation on oocyte's quality in PCOS patients : A double blind trial. Eur Rev Med Pharm Sci. 2011;15:509-14.

31. Lesoine B, Regidor PA. Prospective randomized study on the influence of myo-inositol in PCOS women undergoing IVF in the improvement of oocyte quality, fertilization rate, and embryo quality. Int J Endocrinol. 2016;2016:4378507.

\section{Publisher's Note}

Springer Nature remains neutral with regard to jurisdictional claims in published maps and institutional affiliations.
Ready to submit your research? Choose BMC and benefit from:

- fast, convenient online submission

- thorough peer review by experienced researchers in your field

- rapid publication on acceptance

- support for research data, including large and complex data types

- gold Open Access which fosters wider collaboration and increased citations

- maximum visibility for your research: over $100 \mathrm{M}$ website views per year

At BMC, research is always in progress.

Learn more biomedcentral.com/submissions 